TRANSACTIONS OF THE

AMERICAN MATHEMATICAL SOCIETY

Volume 352, Number 8, Pages 3639-3650

S 0002-9947(00)02467-3

Article electronically published on March 15, 2000

\title{
THE GEOGRAPHY PROBLEM FOR IRREDUCIBLE SPIN FOUR-MANIFOLDS
}

\author{
B. DOUG PARK AND ZOLTÁN SZABÓ
}

\begin{abstract}
We study the geography problem for smooth irreducible simplyconnected spin four-manifolds. For a large class of homotopy types, we exhibit both symplectic and non-symplectic representatives. We also compute the Seiberg-Witten invariants of all the four-manifolds we construct.
\end{abstract}

\section{INTRODUCTION}

In this paper we will work with smooth closed simply-connected 4-manifolds. Such a 4-manifold $X$ is called irreducible, if each connected sum decomposition of $X$ as $X=Y \# Z$ satisfies that either $Y$ or $Z$ is a homotopy $S^{4}$. Clearly each smooth closed simply-connected 4-manifold $X$ can be decomposed as the connected sum of irreducible ones. (Note that this decomposition is not unique, for example $\left(S^{2} \times S^{2}\right) \# \overline{\mathbf{C P}}^{2}=\mathbf{C P}^{2} \# 2 \overline{\mathbf{C P}}^{2}$.)

In their paper [FS1], Fintushel and Stern asked which homotopy types are represented by irreducible simply-connected four-manifolds. Their question can be billed as the topological version of the classical geography problem concerning complex surfaces in algebraic geometry. Clearly this problem is an important step towards the desired classification of simply-connected smooth closed 4-manifolds.

Note that the homotopy type of a smooth closed simply-connected 4-manifold $X$ is determined by its Euler characteristic $e(X)$, its signature $\operatorname{sign}(X)$ and the parity of its intersection form $Q_{X}: H_{2}(X) \otimes H_{2}(X) \rightarrow \mathbf{Z}$. (This is easy to prove in case $Q_{X}$ is nondefinite, and the definite case follows from a famous theorem of Donaldson, see [D.) Clearly $e(X)=2+\operatorname{rank}\left(Q_{X}\right)$ and $\operatorname{sign}(X)=\operatorname{sign}\left(Q_{X}\right)$. Note also that $Q_{X}$ is even if and only if $X$ is a spin manifold. P.

The geography problem for odd intersection forms was studied in [FS1], [St], and

In this paper we will restrict ourselves to the even case. By reversing the orientation of the smooth spin 4-manifold if neccesary, we can assume that $\operatorname{sign}(X) \leq 0$. Rokhlin's theorem states, that $\operatorname{sign}(X)$ is divisible by 16 , and it follows that the intersection form $Q_{X}$ is isomorphic to $k H \oplus 2 n E_{8}$, where $k \geq 0, n \geq 0$, $H=\left(\begin{array}{ll}0 & 1 \\ 1 & 0\end{array}\right)$, and $E_{8}$ is the unique $8 \times 8$ even negative definite unimodular matrix. Let $\mathcal{A}$ denote the set of the intersection forms of the form $k H \oplus 2 n E_{8}$.

Our first result is the following:

Received by the editors April 1, 1998.

2000 Mathematics Subject Classification. Primary 57R15, 57R57; Secondary 57N65, 58D27.

Key words and phrases. Geography, spin, symplectic, Seiberg-Witten.

The second author was supported in part by NSF Grant DMS-970435. 
Theorem 1.1. For each intersection form $Q \in \mathcal{A}$, that satisfies

(i) $2 e(Q)+3 \operatorname{sign}(Q) \geq 0$,

(ii) $e(Q)-\operatorname{sign}(Q) \equiv 0(\bmod 4)$, i.e. $b_{2}^{+}(Q)$ is odd,

(iii) $\operatorname{sign}(Q)<0$,

there is a simply-connected, smooth, irreducible, spin 4-manifold $M$ with $Q_{M}=Q$.

Remark 1.2. (i) It is a very deep open question whether there exists an irreducible simply connected closed smooth spin 4-manifold $X$ with $2 e\left(Q_{X}\right)+3 \operatorname{sign}\left(Q_{X}\right)<0$. This problem is usually called the $3 / 2$ Conjecture; cf. [Ki, Problem 4.93 on p. 302]. Note that a negative answer for this existence problem would imply also the famous $11 / 8$ Conjecture.

(ii) The next assumption on the parity of $b_{2}^{+}$is also important. Note that for a spin 4 -manifold $b_{2}^{+} \equiv b_{2}^{-}(\bmod 2)$. While there are proposed counterexamples for irreducible simply-connected 4 -manifolds with both $b_{2}^{+}$and $b_{2}^{-}$even, see [Ki] (Problem 4.97(B) on p. 304), the available techniques are not sufficient to prove irreducibility since the parity implies that the Seiberg-Witten and Donaldson invariants vanish with both orientation.

(iii) It still remains to consider the $\operatorname{sign}(X)=0$ case. The examples with $b_{2}^{+}$odd are all homotopy equivalent to $(2 n+1)\left(S^{2} \times S^{2}\right)$. It would certainly be great to find irreducible simply-connected four-manifolds homotopy equivalent to $3\left(S^{2} \times S^{2}\right)$ or $5\left(S^{2} \times S^{2}\right)$. While this seems still out of reach, the second author recently was able to construct examples for all $n$ large enough; see [Sz3].

To construct our manifolds we shall start out with some examples of spin symplectic four-manifolds, regarding them as building blocks, and then make repeated usage of the symplectic sum operation invented 1 by Gompf in [G2]. The endproducts of the symplectic sum operations will again be spin and symplectic, and hence they must have nonzero Seiberg-Witten invariants by a theorem of Taubes; see T1]. If they were not irreducible, then in each case we could split off a nontrivial summand which would be forced to have a negative definite intersection form. But by a theorem of Donaldson, see [D], the negative definite intersection form of the summand is diagonalizable and thus odd, contradicting the fact that the summand is spin and hence even. Therefore the results of our construction will all be irreducible.

In the light of the recent counterexamples to the Minimal Conjecture, see [Sz1, and also [Sz2, [FS2], it is natural to ask whether the above homotopy types can be represented by irreducible non-symplectic 4-manifolds. We prove the following result.

Theorem 1.3. For every quadratic form $Q$ described in Theorem 1.1, there is a simply-connected irreducible 4-manifold $X$ with $Q_{X}=Q$, such that $X$ doesn't admit symplectic structures with either orientation.

In Section 2 we present the main ideas of the construction and examples with $\operatorname{sign}(X)=-32$. Theorem 1.1 is proved in Section 3. Other interesting examples are presented in Section 4 The computation of Seiberg-Witten invariants and the proof of Theorem [1.3 are given in the last section.

\footnotetext{
${ }^{1}$ The referee has kindly pointed out to the authors that the symplectic sum operation was first mentioned in Gromov's book $\mathrm{Gr}$.
} 


\section{EXAMPLES With SIGNATURE -32}

In this section we will construct simply-connected, symplectic, irreducible, spin, 4-manifolds $X_{k}$ with $Q_{X_{k}}=(2 k+1) H \oplus 4 E_{8}$, for all integers $k \geq 3$, i.e. $\operatorname{sign}\left(X_{k}\right)=$ $-32, e\left(X_{k}\right)=36+4 k$.

Our first building block will be the fiber sum of two copies of the KodairaThurston manifold, (cf. G2, p. 570]). Let $(a, b)$ be the generators of $\pi_{1}\left(T^{2}\right)$, and $\varphi: T^{2} \rightarrow T^{2}$ a diffeomorphism which maps $(a, b)$ to $(a b, b)$. Let $Y$ be the mapping torus of $\varphi$, i.e., a $T^{2}$ bundle over $S^{1}$ with monodromy $\varphi$. The Kodaira-Thurston manifold $Z$ is defined to be the product $S^{1} \times Y$. $Z$ fibers over $T^{2}$ with $T^{2}$ fiber, with trivial monodromy around the first factor, and $Z$ has a section of square 0 . Now take two copies $Z$ and $Z^{\prime}$, and fix fibers $F \subset Z$ and $F^{\prime} \subset Z^{\prime}$. Let $f: F \rightarrow F^{\prime}$ be a diffeomorphism which maps $(a, b)$ into $\left(-b^{\prime}, a^{\prime}\right)$. Let $W$ denote the induced fiber sum $Z \sharp_{f} Z^{\prime}$. Note that $W$ fibers over a genus 2 surface and the fibration admits a section $\Sigma$ of square 0 , which is gotten by pasting together the sections of $Z$ and $Z^{\prime}$. Now $W$ has a symplectic structure and $\Sigma$ is a symplectic submanifold (cf. [Th, and [G2]).

Now suppose that $X$ is a symplectic 4-manifold, and $\Sigma_{2} \hookrightarrow X$ is a symplectic submanifold with $g\left(\Sigma_{2}\right)=2$ and self-intersection 0 . Then we can form the symplectic sum of $W$ and $X$ along $\Sigma$ and $\Sigma_{2}$. We recall the symplectic sum operation in greater length below, cf. [G2].

Let $\nu_{1}$ and $\nu_{2}$ be the normal bundles of $\Sigma$ and $\Sigma_{2}$ in $T W$ and $T X$, respectively. Since $e\left(\nu_{1}\right)=-e\left(\nu_{2}\right)=0$ and $g(\Sigma)=g\left(\Sigma_{2}\right)$, we can fix a fiber-orientation reversing bundle isomorphism $g: \nu_{1} \rightarrow \nu_{2}$. We identify the tubular neighborhoods $N_{1}$ of $\Sigma$ and $N_{2}$ of $\Sigma_{2}$ with the open disk bundles of radius $\pi^{-1 / 2}$ in $\nu_{1}$ and $\nu_{2}$. Let $\imath$ map $N_{2}$ minus the 0 -section into itself by

$$
\imath(x)=\left(\frac{1}{\pi\|x\|^{2}}-1\right)^{\frac{1}{2}} x
$$

on each punctured fiber. Note that $\imath$ turns each punctured normal disk inside out. We obtain a fiber-orientation preserving diffeomorphism $\psi=\imath \circ g: N_{1}-\Sigma \rightarrow$ $N_{2}-\Sigma_{2}$.

Let

$$
W \#_{g} X=(W-\Sigma) \cup_{\psi}\left(X-\Sigma_{2}\right)
$$

where we use $\psi$ to identify $N_{1}-\Sigma$ and $N_{2}-\Sigma_{2}$. W\# ${ }_{g} X$ is smooth, closed, oriented manifold. By [G2], $W \#_{g} X$ admits canonical symplectic structure, which is obtained from the corresponding symplectic structures of $W$ and $X$.

Lemma 2.1. Let $W, X, \Sigma, \Sigma_{2}$ be as above, and suppose that $\pi_{1}\left(X-\Sigma_{2}\right)=1$. Then $W \#_{g} X$ is a simply-connected symplectic 4-manifold with $e\left(W \#_{g} X\right)=e(X)+4$ and $\operatorname{sign}\left(W \#_{g} X\right)=\operatorname{sign}(X)$. Furthermore if $X$ is a spin manifold, then $g$ can be chosen in such a way that $W \#_{g} X$ is also spin.

Proof. It can be shown (cf. G2, p. 571]) that $\pi_{1}(W-\Sigma) /\left\langle\pi_{1}\left(\Sigma^{\|}\right)\right\rangle=1$, where $\Sigma^{\|}$ is a parallel copy of $\Sigma$ in $W-\Sigma$. Since $\Sigma$ gets identified with $\Sigma_{2}$ in $X$ and the inclusion $\Sigma^{\|} \hookrightarrow X-\Sigma_{2}$ induces the zero map on the fundamental groups, an easy application of van Kampen's theorem gives $\pi_{1}\left(W \#_{g} X\right)=1$. Various topological invariants behave nicely under the symplectic sum operation (cf. G2, p. 535] for 
the general formulae):

$$
\begin{gathered}
\operatorname{sign}\left(W \#_{g} X\right)=\operatorname{sign}(W)+\operatorname{sign}(X)=\operatorname{sign}(X), \\
e\left(W \#_{g} X\right)=e(W)+e(X)-2 e(\Sigma)=e(X)+4 .
\end{gathered}
$$

The last assertion about the spin structure is a special case of Proposition 1.2 in G2] (p. 536).

We can iterate the above construction. Namely, we take $n$ copies of $W$, labelled $W_{1}, \ldots, W_{n}$, and for each $j=1, \ldots, n$ we fix a copy of $\Sigma, \Sigma^{(j)} \subset W_{j}$. Take $X$ and choose $n$ parallel copies of $\Sigma_{2}$ inside it, labelled $\Sigma_{2}^{\prime}, \Sigma_{2}^{\prime \prime}, \ldots, \Sigma_{2}^{(n)}$. Successively glue each $W_{j}$ onto the fixed $X$ by symplectically summing along $\Sigma^{(j)}=\Sigma_{2}^{(j)}$. Let

$$
X \#_{g} n W=\left(\coprod_{j}\left(W_{j}-\Sigma^{(j)}\right)\right) \bigcup_{\left(\psi^{\prime}, \ldots, \psi^{(n)}\right)}\left(X-\bigcup_{j} \Sigma_{2}^{(j)}\right) .
$$

As in the case $n=1$, we have

Lemma 2.2. Suppose that $\pi_{1}\left(X-\Sigma_{2}\right)=1$. Then $X \#_{g} n W$ is a simply-connected, symplectic 4-manifold with $e\left(X \#_{g} n W\right)=e(X)+4 n$, and $\operatorname{sign}\left(X \#_{g} n W\right)=\operatorname{sign}(X)$. Furthermore if $X$ is a spin manifold, then $g$ can be chosen in such a way that $X \#_{g} n W$ is also spin.

Proof. Note that the surface $\Sigma^{\|}$lying in any $W_{j}-\Sigma^{(j)}$ summand is isotopic to another parallel surface in $X-\left(\Sigma_{2} \times D^{2}\right)$. Hence the inclusion $\Sigma^{\|} \hookrightarrow X-\bigcup_{j} \Sigma_{2}^{(j)}$ induces the zero map on $\pi_{1}$. As in the proof of Lemma 2.1, this implies that any loop in $W_{j}-\Sigma^{(j)}$ summand is trivial in $\pi_{1}\left(X \#_{g} n W\right)$. Since $\pi_{1}\left(X-\bigcup_{j} \Sigma_{2}^{(j)}\right)$ is generated by the meridians of the surfaces $\Sigma_{2}^{(j)}$, which can be pushed off into the corresponding $W_{j}-\Sigma^{(j)}$ summand, $X \#_{g} n W$ is simply connected. $e\left(X \#_{g} n W\right)=$ $e(X)+n(e(W))-2 n(e(\Sigma))$ and $\operatorname{sign}\left(X \#{ }_{g} n W\right)=\operatorname{sign}(X)+n(\operatorname{sign}(W))$. The last assertion follows immediately from the previous lemma.

Let $V_{n}$ denote a simply-connected minimal elliptic surface without multiple fibers, and with geometric genus $p_{g}=n-1$. Note that $\operatorname{sign}\left(V_{n}\right)=-8 n$ and $e\left(V_{n}\right)=12 n$ (cf. G1] $)$.

To obtain our examples $X_{k}$, we apply the above lemmas by setting $X=V_{4}$. We recall that $V_{4}$ is a spin manifold with the intersection form $7 H \oplus 4 E_{8}$. Also recall that there is a holomorphic projection $p: V_{4} \rightarrow S^{2}$ with the generic fiber $F=T^{2}$, a torus. The projection admits a section $S$, i.e., an embedding $S^{2} \rightarrow V_{4}$, which transversely intersects every fiber exactly once. The normal Euler class of $S$ is -4 . Now take two distinct torus fibers and a copy of the section $S$. We can symplectically resolve the two intersection points and get a genus 2 surface $\Sigma_{2}$. Note that $\left[\Sigma_{2}\right] \cdot\left[\Sigma_{2}\right]=([S]+2[F]) \cdot([S]+2[F])=0$, since $[F] \cdot[F]=0$.

Next we observe that $\pi_{1}\left(V_{4}-\Sigma_{2}\right)=1$. By van Kampen's theorem,

$$
1=\pi_{1}\left(V_{4}\right)=\frac{\pi_{1}\left(V_{4}-\Sigma_{2}\right) * \pi_{1}\left(\Sigma_{2} \times D^{2}\right)}{\pi_{1}\left(\Sigma_{2} \times\left(D^{2}-\{0\}\right)\right)}=\frac{\pi_{1}\left(V_{4}-\Sigma_{2}\right)}{\iota_{*} \pi_{1}\left(p t \times \partial D^{2}\right)},
$$

where $\iota$ denotes the appropriate inclusion. Let $\gamma$ represent a generator of $\pi_{1}\left(p t \times \partial D^{2}\right)$. By requiring the $p t$ to lie on the section part of $\Sigma_{2}$, we can assume without loss of generality that the image of $\iota \circ \gamma$ lies on a fiber $F$. But we know that all the 1 -cycles of $F$ vanish inside $V_{4}$, i.e., $\iota_{*} \pi_{1}(F-p t)=1$ inside $\pi_{1}\left(V_{4}-\Sigma_{2}\right)$. Thus we see that $\pi_{1}\left(V_{4}-\Sigma_{2}\right)=1$. 
We define $X_{3}=V_{4}$ and for $k \geq 4, X_{k}=V_{4} \#_{g}(k-3) W$. We have $\operatorname{sign}\left(X_{k}\right)=-32$ and $e\left(X_{k}\right)=48+4(k-3)=4 k+36$. Note that $X_{k}$ is indefinite and spin, and hence by the above computation of its signature and Euler characteristic the intersection form of $X_{k}$ must be $(2 k+1) H \oplus 4 E_{8}$. Since spin, symplectic four-manifolds are irreducible, we get the desired examples.

\section{Proof of Theorem 1.1}

For starters, we prove the following

Proposition 3.1. For each integer $n \geq 1$, there exists a simply-connected irreducible spin symplectic four-manifold, $M_{n}$, with the intersection form $(2 n+1) H \oplus$ $2 E_{8}$.

Proof. For our second family $M_{n}$, we will work with a homotopy $K 3$ surface in place of $V_{4}$. Recall that a compact complex analytic surface $X$ is called a $K 3$ surface if $\pi_{1}(X)=1$ and $c_{1}(X)=0$. It is a classical result of Kodaira that any two $K 3$ surfaces are diffeomorphic. Note that the elliptic surface $V_{2}$, defined in the previous section, is also a $K 3$ surface. Let $F$ and $S$ denote the regular fiber and the section of the elliptic surface $V_{2}$. Roughly speaking, our homotopy $K 3$ surface will be obtained from $V_{2}$ by removing a tubular neighborhood of a torus fiber $F$ and replacing it with the complement of the trefoil knot in $S^{3}$ crossed with $S^{1}$. This construction is a special case of the powerful machinery developed by Fintushel and Stern in [FS2.

Let $K$ denote the trefoil knot in $S^{3}$ and $m$ a meridional circle to $K$. Perform the 0 -framed surgery on $K$ and call the resulting 3-manifold by $S^{3}(K)$. In $S^{3}(K) \times S^{1}$ we have the smoothly embedded torus $T_{m}=m \times S^{1}$ of self-intersection 0 . Since a neighborhood of $m$ has a canonical framing in $S^{3}(K)$, a neighborhood of the torus $T_{m}$ in $S^{3}(K) \times S^{1}$ has a canonical identification with $T_{m} \times D^{2}$. Let $V_{K}$ denote the fiber sum

$V_{K}=V_{2} \#_{F=T_{m}}\left(S^{3}(K) \times S^{1}\right)=\left[V_{2}-\left(F \times D^{2}\right)\right] \cup_{\psi}\left[\left(S^{3}(K) \times S^{1}\right)-\left(T_{m} \times D^{2}\right)\right]$,

where the two pieces are glued together so as to preserve the homology class $\alpha=$ $\left[p t \times \partial D^{2}\right]$. Fintushel and Stern showed that $V_{K}$ is homotopy equivalent to $V_{2}$, i.e., $V_{K}$ is a homotopy $K 3$ surface. Moreover, using Thurston's theorem [Th] and the fact that $K$ is a fibered knot with a punctured torus as a fiber, they showed that $V_{K}$ is in fact symplectic.

Recall that the Seifert surface $\Sigma^{0}$ of the trefoil knot $K$ is a punctured torus. Since the gluing map $\psi$ sends the homology class $\alpha$ into the homology class $[K \times$ $p t]=\left[\partial \Sigma^{0} \times p t\right]$ in $\left(S^{3}(K) \times S^{1}\right)-\left(T_{m} \times D^{2}\right)$, we can glue together $\Sigma^{0}$ and the punctured section $S-\left(F \times D^{2}\right)$, and get a smooth torus $\Sigma_{1}$ in $V_{K}$. Note that $\left[\Sigma_{1}\right] \cdot\left[\Sigma_{1}\right]=[S] \cdot[S]=-2$, and $[F] \cdot\left[\Sigma_{1}\right]=[F] \cdot[S]=1$. As before, we resolve the intersection of $F$ and $\Sigma_{1}$, and get a smooth genus 2 surface $\Sigma_{2}$ with self-intersection $\left[\Sigma_{2}\right] \cdot\left[\Sigma_{2}\right]=\left([F]+\left[\Sigma_{1}\right]\right) \cdot\left([F]+\left[\Sigma_{1}\right]\right)=0$. Gompf's construction tells us that $\Sigma_{1}$ is a symplectic submanifold of $V_{K}$ (since $\Sigma_{1}$ is the symplectic sum of the symplectic section $S$ in $V_{2}$ and the symplectic fiber $\overline{\Sigma^{0}}$ of $\left.S^{3}(K) \times S^{1}\right)$. It follows that $\Sigma_{2}$ is a symplectic surface in $V_{K}$.

Since $V_{K}$ is actually homeomorphic to $V_{2}$, we have $\pi_{1}\left(V_{K}\right)=1$. We claim that $\pi_{1}\left(V_{K}-\Sigma_{2}\right)=1$. By van Kampen's theorem,

$$
1=\pi_{1}\left(V_{K}\right)=\frac{\pi_{1}\left(V_{K}-\Sigma_{2}\right) * \pi_{1}\left(\Sigma_{2} \times D^{2}\right)}{\pi_{1}\left(\Sigma_{2} \times\left(D^{2}-\{0\}\right)\right)}=\frac{\pi_{1}\left(V_{K}-\Sigma_{2}\right)}{\iota_{*} \pi_{1}\left(p t \times \partial D^{2}\right)}
$$


where $\iota$ denotes the appropriate inclusion. By choosing the $p t$ to lie on the $S$-part of $\Sigma_{2}$, we can assume that $\iota$ maps a loop representing the generator of $\pi_{1}\left(p t \times \partial D^{2}\right)$ into a fiber $F$ inside the $V_{2}$-part of $V_{K}$. Since all the loops on $F$ are homotopically trivial inside a neighborhood of $F$ in $V_{2}$, we conclude that $\iota_{*}$ is the trivial map. Hence $\pi_{1}\left(V_{K}-\Sigma_{2}\right)=1$.

Now we use Lemmas 2.1 and 2.2 of the previous section. For $n=1$, let $M_{1}=$ $V_{K}=X$. For $n \geq 2$, let $M_{n}=V_{K} \#_{g}(n-1) W$. For every $n \geq 1, M_{n}$ is a spin, symplectic four-manifold hence irreducible. Since $M_{n}$ is indefinite and $\operatorname{sign}\left(M_{n}\right)=$ $\operatorname{sign}\left(V_{K}\right)=-16, e\left(M_{n}\right)=24+4(n-1)=4 n+20$, the intersection form of $M_{n}$ is $(2 n+1) H \oplus 2 E_{8}$.

In order to finish the proof of Theorem 1.1 we will use a result of [GM]. Let us recall that the Gompf nucleus of the $K 3$ surface is the regular neighborhood of the union of a cusp fiber and a section. Let $\mathcal{K}$ denote a $K 3$ surface. It is proved in GM that $\mathcal{K}$ contains 3 disjoint nuclei, each corresponding to a different Kummer surface description of $\mathcal{K}$. (Essentially the three nuclei correspond to three different coordinate projections of $T^{4} \rightarrow T^{2}$.) Using the concrete coordinate description of the three nuclei in GM] (p. 109), we can easily find a symplectic form on $\mathcal{K}$ such that the regular fiber and section of one nucleus and the regular fiber of another nucleus are symplectic submanifolds of $\mathcal{K}$, cf. $\left[\mathrm{MSz}\right.$. Now choose two nuclei, $N^{\prime}$ and $N^{\prime \prime}$, and fix such a symplectic structure on $\mathcal{K}$. We denote the torus fiber and the section of $N^{\prime}$ by $F^{\prime}$ and $S^{\prime}$, etc. Inside $N^{\prime}$, there is a symplectic torus $\Sigma_{1}^{\prime}$ of square 0 such that $\left[\Sigma_{1}^{\prime}\right]=\left[F^{\prime}\right]+\left[S^{\prime}\right]$ in $H_{2}(\mathcal{K})$. We do the Fintushel-Stern construction at the torus fiber $F^{\prime}$ with the trefoil knot $K$ in $S^{3}$. We get $\mathcal{K}_{K}=\mathcal{K} \#_{F^{\prime}=T_{m}}\left(S^{3}(K) \times S^{1}\right)$. As in the construction of $M_{n}$, we can glue $\Sigma_{1}^{\prime}$ and the Seifert surface of $K$ to get a genus 2 symplectic surface $\Sigma_{2}$ of square 0 , embedded inside $\mathcal{K}_{K}$. Next define $M(0)=\mathcal{K}_{K}$, and let

$$
M(s)=\mathcal{K}_{K} \#_{F^{\prime \prime}=F} V_{2 s}
$$

be the fiber connected sum of $\mathcal{K}_{K}$ and $V_{2 s}$ along the tori $F^{\prime \prime}$ and $F$, where $F^{\prime \prime}$ is a torus fiber in $N^{\prime \prime} \subset \mathcal{K}_{K}$ and $F$ is a torus fiber inside $V_{2 s}(s \geq 1)$. Now we can use Lemmas 2.1 and 2.2 with $X=M(s)$. We denote the results by

$$
M_{n}(s)=M(s) \#_{g} n W .
$$

Note that $M_{n}(0)$ is diffeomorphic to $M_{n+1}$. Also we have $\operatorname{sign}\left(M_{n}(s)\right)=\operatorname{sign}(M(s))$ $=-16(s+1)$, and $e\left(M_{n}(s)\right)=24(s+1)+4 n$. We have thus proved the following

Proposition 3.2. For each integer $s \geq 0$ and $n \geq 0$, there is a simply-connected, irreducible, spin, symplectic four-manifold, $M_{n}(s)$, with the intersection form $(4 s+3+2 n) H \oplus 2(s+1) E_{8}$.

Now Theorem 1.1 follows from Proposition 3.2 .

\section{More FAMILIES}

Our construction can be slightly modified to produce other irreducible spin manifolds of signature $(-16) s$, for $s \geq 3$. It will turn out in the next section that the families constructed here have much simpler Seiberg-Witten invariants than $M_{n}(s)$. Let us first discuss the signature -48 case $(s=3)$ in some detail. The central building block in this case will be the elliptic surface $V_{6}$. Recall that $\operatorname{sign}\left(V_{6}\right)=-8 \cdot 6=-48, e\left(V_{6}\right)=12 \cdot 6=72$, and $Q_{V_{6}}=11 H \oplus 6 E_{8}$. If $F$ and $S$ denote the regular torus fiber and the section of $V_{6}$, then there is a genus 
3 surface $\Sigma_{3} \subset V_{6}$ with $\left[\Sigma_{3}\right]=3[F]+[S]$ in $H_{2}\left(V_{6}\right)$ (gotten by resolving the three intersection points). Since $[S]^{2}=-6$, we have $\left[\Sigma_{3}\right]^{2}=0$. The second building block (that replaces $W$ ) is gotten by applying the techniques in [FS2] to the torus fiber $T$ in $W$. Let $W_{K}=W \#_{T=T_{m}}\left(S^{3}(K) \times S^{1}\right)$, where $K$ is the trefoil knot (cf. Section 3 and [FS2]). If $\Sigma^{0}$ denotes the Seifert surface of $K$, then we can glue $\Sigma^{0}$ and the section of $W$ along the meridional circle of $T_{m}=T$. The result is a genus 3 surface $\Xi_{3}$ of square 0 . Since $W_{K}$ has the same homology and intersection pairing as $W$, we have $\operatorname{sign}\left(W_{K}\right)=e\left(W_{K}\right)=0$.

Now fix a copy of $V_{6}$ and take $q$ copies of $W_{K}(q \geq 0)$. Choose $q$ parallel copies of $\Sigma_{3}$ in $V_{6}$ and successively glue on a copy of $W_{K}$ for each copy of $\Sigma_{3}$, using Gompf's symplectic sum operation to identify the punctured tubular neighborhoods of $\Sigma_{3}$ and $\Xi_{3}$. We denote the result by

$$
Y(3, q)=V_{6} \#_{\Sigma_{3}=\Xi_{3}} q W_{K} .
$$

Note that $\operatorname{sign}(Y(3, q))=\operatorname{sign}\left(V_{6}\right)=-48$, and

$$
e(Y(3, q))=e\left(V_{6}\right)+q\left(e\left(W_{K}\right)\right)-2 q\left(e\left(\Sigma_{3}\right)\right)=72+8 q .
$$

Using the fact that $\pi_{1}\left(V_{6}-\Sigma_{3}\right)=1$, one can easily show that $Y(3, q)$ is simply connected. Since $Y(3, q)$ is spin, $Q_{Y(3, q)}=(11+4 q) H \oplus 6 E_{8}$. As before, $Y(3, q)$ is symplectic by construction and hence irreducible.

Finally we consider the general case $s \geq 3$. The central building block is the elliptic surface $V_{2 s}$. We have $\operatorname{sign}\left(V_{2 s}\right)=-16 s, e\left(V_{2 s}\right)=24 s$, and $Q_{V_{2 s}}=(4 s-$ 1) $H \oplus(2 s) E_{8}$. There is a symplectic genus $s$ surface $\Sigma_{s} \subset V_{2 s}$ such that $\left[\Sigma_{s}\right]=s[F]+$ $[S]$ in $H_{2}\left(V_{2 s}\right)$. The other building block is $W_{(s-2) K}=(s-2)\left(S^{3}(K) \times S^{1}\right) \#_{T_{m}=T} W$, where we remove $(s-2)$ copies of the fiber $T$ from $W$ and glue back $(s-2)$ copies of $\left[\left(S^{3}(K) \times S^{1}\right)-T_{m}\right] \cdot \operatorname{sign}\left(W_{(s-2) K}\right)=e\left(W_{(s-2) K}\right)=0$. In $W_{(s-2) K}$ there is a genus $s$ surface $\Xi_{s}$ of square 0 , gotten by gluing the section of $W$ and $(s-2)$ copies of the Seifert surface $\Sigma^{0}$ (from each copy of $S^{3}(K) \times S^{1}$ ) along $(s-2)$ meridians of $T=T_{m}$. Now using Gompf's symplectic sum operation, we define

$$
\begin{gathered}
Y(s, q)=V_{2 s} \#_{\Sigma_{s}=\Xi_{s}} q W_{(s-2) K} . \\
\operatorname{sign}(Y(s, q))=\operatorname{sign}\left(V_{2 s}\right)=-16 s, \text { and } \\
e(Y(s, q))=e\left(V_{2 s}\right)+q\left(e\left(W_{(s-2) K}\right)\right)-2 q\left(e\left(\Sigma_{s}\right)\right) \\
=24 s-2 q(2-2 s)=24 s+4 q(s-1) .
\end{gathered}
$$

In conclusion, we have the following:

Proposition 4.1. For each integer $s \geq 3$ and $q \geq 0$, the 4-manifold $Y(s, q)$ defined above is a simply-connected spin symplectic 4-manifold with the intersection form $(4 s-1+2 q(s-1)) H \oplus(2 s) E_{8}$.

\section{Seiberg-Witten invariants}

In this section we compute the Seiberg-Witten invariants of every 4-manifold constructed in the previous sections.

Suppose that $X$ is a smooth closed 4-manifold $X$ with $b_{2}^{+}(X)>1$. After fixing a homology orientation of $X$ (i.e., an orientation of $\operatorname{det} H_{+}^{2}(X, \mathbf{R}) \otimes \operatorname{det} H^{1}(X, \mathbf{R})$ ), the Seiberg-Witten invariant is defined on the set of $\operatorname{spin}^{c}$ structures over $X$. In case $H_{1}(X, \mathbf{Z})$ has no 2-torsion, the set of $\operatorname{spin}^{c}$ structures over $X$ can be naturally identified with

$$
\mathcal{C}_{X}=\left\{L \in H^{2}(X, \mathbf{Z}) \mid L \equiv w_{2}(T X) \quad(\bmod 2)\right\},
$$


and we can view the Seiberg-Witten invariant as

$$
S W_{X}: \mathcal{C}_{X} \rightarrow \mathbf{Z}
$$

An $L \in \mathcal{C}_{X}$ is called a Seiberg-Witten basic class if $S W_{X}(L) \neq 0$. For more on the basics of Seiberg-Witten invariants, see [KM], [M], and [Wi].

The main ingredients in our computations will be a result of Taubes on symplectic 4-manifolds on one hand, and the adjunction inequality for Seiberg-Witten invariants on the other. Let us recall them below.

Proposition 5.1 (see [T1]). Let $X$ be a closed symplectic 4-manifold with $b_{2}^{+}(X)$ $>1$. Let $c_{1}(X)$ denote the canonical class of the symplectic structure. Then

$$
S W_{X}\left(c_{1}(X)\right)= \pm 1 \text {. }
$$

Proposition 5.2. Adjunction inequality (see [KM], MST]). Suppose that $\Sigma \hookrightarrow$ $X$ is a smoothly embedded surface of genus $g(\Sigma)>0$ with self-intersection $[\Sigma]^{2} \geq 0$. Then for every basic class $L$ of $X$ we have

$$
2 g(\Sigma)-2 \geq|\langle L,[\Sigma]\rangle|+[\Sigma] \cdot[\Sigma] .
$$

Now we compute $S W_{X_{k}}$. First note that $X_{3}=V_{4}$ and the Seiberg-Witten invariants of elliptic surfaces are known, see [FS3]. We have $S W_{V_{4}}( \pm 2 P D(F))=1$, and $S W_{V_{4}}(0)=-2$, where $F$ is the homology class of the fiber. Furthermore $S W_{V_{4}}(L)=0$, for all $L \neq 0, \pm 2 P D(F)$.

For $k \geq 4$, we prove the following.

Theorem 5.3. For each $k \geq 4$, let $X_{k}$ denote the symplectic 4-manifold defined

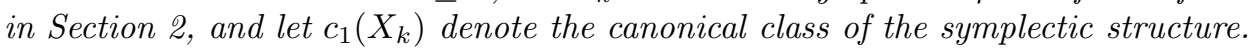
Then

$$
S W_{X_{k}}\left( \pm c_{1}\left(X_{k}\right)\right)= \pm 1
$$

and $S W_{X_{k}}(L)=0$ for all $L \neq \pm c_{1}\left(X_{k}\right)$.

Proof. If $k=4$, then $X_{4}=W \#_{g} V_{4}$, and the intersection form of $X_{4}$ is $9 H \oplus 4 E_{8}$. Let $L$ be a basic class of $X_{4}$. In order to determine $L$, we decompose the intersection form into four parts, namely,

$$
4 E_{8}, 2 H \oplus 2 H \oplus 2 H, H \oplus H, H .
$$

Let us describe this decomposition below. The $4 E_{8}$ part comes from the $V_{4}$ summand inside $X_{4}$. One can find a basis for the corresponding subspace of $H_{2}\left(X_{4}\right)$ consisting solely of 2 -spheres with self-intersection -2 . There is a clever trick due to Stipsicz that allows one to conclude that $L$ is orthogonal to these 2-spheres (cf. St]). The $2 H \oplus 2 H \oplus 2 H$ part also comes from the $V_{4}$ summand of $X_{4}$. Recall that $V_{4}=V_{1} \sharp_{f} V_{1} \sharp_{f} V_{1} \sharp_{f} V_{1}$, where $\sharp_{f}$ denotes the fiber connected sum operation. Each $2 H$ occuring in this part corresponds to a single $\sharp_{f}$-operation in $V_{4}$. For each $2 H$, one can find a basis for the corresponding subspace of $H_{2}\left(X_{4}\right)$ consisting solely of tori with self-intersection 0 . Hence by the adjunction inequality the basic class $L$ is orthogonal to these tori. The $H \oplus H$ part comes from the $W$ summand of $X_{4}$. Recall that there is a basis of $H_{2}(W) \cong \mathbf{Z}^{6}$ consisting of four tori, a fiber, and the section $\Sigma$ (cf. [Sz4 p. 413]). The four tori in $W$ (which all have square 0 and are disjoint from a generic fiber and section in $W$ ) comprise a basis for the 
corresponding $H \oplus H$ part of $H_{2}\left(X_{4}\right)$. So again by the adjunction inequality, $L$ is orthogonal to these tori.

Finally the $H$ part comes from the gluing area of the symplectic sum operation $\#_{g}$. Choose a meridian $\mu$ of $\Sigma=\Sigma_{2}$. We can cut and then glue the torus fibers of $V_{4}$ and $W$ along $\mu$ to obtain a symplectic genus 2 surface $F_{2}$. Note that $\left[F_{2}\right] \cdot\left[F_{2}\right]=0$ and $\left[F_{2}\right] \cdot\left[\Sigma_{2}\right]=1$. A basis for the corresponding subspace of $H_{2}\left(X_{4}\right)$ is $\left\{\left[F_{2}\right],\left[\Sigma_{2}\right]\right\}$. By the above considerations, $L=P D\left(n\left[F_{2}\right]+m\left[\Sigma_{2}\right]\right)$. Since $\Sigma_{2}$ has genus 2 and self-intersection 0 , the adjunction inequality implies $2 \geq|n|$. Since $F_{2}$ has genus 2 and self-intersection 0 , we have $2 \geq|m|$. Since $L$ is an integral lift of $w_{2}\left(X_{4}\right)$, it follows that $n=L \cdot\left[\Sigma_{2}\right] \equiv\left[\Sigma_{2}\right] \cdot\left[\Sigma_{2}\right]=0(\bmod 2)$, whence $n=0, \pm 2$. Now recall that by definition of the Seiberg-Witten invariant for every basic class $L$ we have $L^{2} \geq 3 \operatorname{sign}\left(X_{4}\right)+2 e\left(X_{4}\right)$. Thus $2 n m \geq 3(-32)+2 \cdot 52=8$, which implies $L= \pm P D\left(2\left[F_{2}\right]+2\left[\Sigma_{2}\right]\right)$. (The last inequality also gives an alternative proof for the fact that $L$ is orthogonal to the $4 E_{8}$ part.) Now the $k=4$ case follows from the theorem of Taubes.

For $k=5$, the intersection form $Q_{X_{5}}=(H \oplus H) \oplus Q_{X_{4}}$, where the new $H \oplus H$ summand comes from the second copy of $W$ that is glued onto $V_{4}$. As before, the $H \oplus H$ part has a basis consisting of four tori of self-intersection 0 that are found inside the second copy of $W$. The first three parts of the argument for $k=4$ go through without a change, and we conclude that $L$ is orthogonal to a codimension 2 subspace of $H_{2}\left(X_{5}\right)$. Note that $X_{5}$ is the result of two symplectic sum operations along two parallel copies of $\Sigma_{2}$ in $V_{4}$ so that we can find a torus fiber $F$ of $V_{4}$ which has two disjoint disks cut out along the meridians of the two copies of $\Sigma_{2}$. Onto each hole of this $F$, we glue an appropriate torus fiber, minus the meridional disk of $\Sigma$, from the corresponding copy of $W$. These three tori are glued together to form a symplectic genus 3 surface $F_{3}$. Note that $\left[F_{3}\right] \cdot\left[F_{3}\right]=0$ and $\left[F_{3}\right] \cdot\left[\Sigma_{2}\right]=1$. We must have $L=P D\left(n\left[F_{3}\right]+m\left[\Sigma_{2}\right]\right)$. Again, by the adjunction inequality, $n=0, \pm 2$ and $4 \geq|m|$. Since $2 m n=L^{2} \geq 3 \operatorname{sign}\left(X_{5}\right)+2 e\left(X_{5}\right)=16$, we conclude that $L= \pm P D\left(2\left[F_{3}\right]+4\left[\Sigma_{2}\right]\right)$.

Now we see the general trend. For $k \geq 6$, the same argument as above shows that $X_{k}$ has only two Seiberg-Witten basic classes, $\pm P D\left(2\left[F_{k-2}\right]+2(k-3)\left[\Sigma_{2}\right]\right)$, where $F_{k-2}$ is a symplectic genus $(k-2)$ surface gotten by gluing one copy of the fiber $F$ of $V_{4}$ and $(k-3)$ copies of the fiber from $(k-3)$ copies of $W$ along the meridional circles of $\Sigma=\Sigma_{2}$. For these classes the theorem of Taubes shows that the Seiberg-Witten invariant is equal to \pm 1 , and this finishes the proof of Theorem 5.3 .

Having disposed of $X_{k}$, we now proceed to determine the Seiberg-Witten basic classes of $M_{n}$. For $n=1, M_{1}=V_{K}$ and it is shown in [FS2] that the only basic classes are $0, \pm 2 P D[F]$, and for a given homology orientation $S W_{V_{K}}(0)=1$, $S W_{V_{K}}( \pm 2 P D[F])=-1$. For $n \geq 2$, we prove the following.

Theorem 5.4. For each $n \geq 2$, let $M_{n}$ denote the symplectic 4-manifold defined in Section 3 Then $S W_{M_{n}}\left( \pm c_{1}\left(M_{n}\right)\right)= \pm 1$, and for all $L \neq \pm c_{1}\left(M_{n}\right)$ we have $S W_{M_{n}}(L)=0$.

Proof. Suppose that $n=2$, and let $\mathcal{H} \subset H_{2}\left(M_{2}, \mathbf{R}\right)$ be the linear subset spanned by $\left[F_{2}\right],\left[\Sigma_{2}\right]$, where $F_{2}$ is the genus 2 symplectic surface gotten by gluing the torus fibers of $V_{2}$ (sitting inside $V_{K}$ ) and $W$ along a vertical meridian of $\Sigma=\Sigma_{2}$. A straightforward argument as in the above proof shows that each basic class $L$ lies 
in $\mathcal{H}$. Now using the adjunction inequality for $F_{2}$ and $\Sigma_{2}$, and also the inequality $L^{2} \geq 2 e\left(M_{2}\right)+3 \operatorname{sign}\left(M_{2}\right)$ we get that $L= \pm P D\left(2\left[F_{2}\right]+2\left[\Sigma_{2}\right]\right)$.

The same argument works also for $n \geq 3$. In this case we get that the only basic classes are $\pm\left(2\left[F_{n}\right]+2(n-1)\left[\Sigma_{2}\right]\right)$, where $F_{n}$ is a genus $n$ symplectic surface gotten by gluing a copy of regular torus fiber $F$ of $V_{2}$ inside $V_{K}$ with $(n-1)$ copies of torus fibers from $(n-1)$ copies of $W$ along the meridians of $(n-1)$ copies of $\Sigma=\Sigma_{2}$. Now Theorem 5.4 follows from the theorem of Taubes.

Next we determine the SW-basic classes of $M_{n}(s)$ when $s \geq 1$. Let $n=0$. Then by definition $M_{0}(s)=M(s)$ and it follows from [FS2], that each basic class $L$ satisfies that $L=a P D\left[F^{\prime}\right]+c P D[F]$, where $a=0, \pm 2$ and $c=0, \pm 2, \ldots, \pm 2 s$, $F^{\prime}$ is a torus fiber in $N^{\prime} \subset \mathcal{K}_{K}$, and $F$ is a regular fiber of $V_{2 s}$. Furthermore

$$
S W_{M(s)}\left(P D\left(a\left[F^{\prime}\right]+c[F]\right)\right)= \pm\left(\begin{array}{c}
2 s \\
s+c / 2
\end{array}\right) .
$$

Now suppose $n>0$. Let $\Gamma_{n+1}$ denote a genus $(n+1)$ surface in $M_{n}(s)$, gotten by gluing a copy of $F^{\prime}$ and $n$ copies of the fiber $T$ of $W$ along the $n$ parallel meridional circles of $\Sigma_{2}$. We have $\left[\Gamma_{n+1}\right]^{2}=0$ and $\left[\Gamma_{n+1}\right] \cdot\left[\Sigma_{2}\right]=1$. Let $\Theta$ denote the 2sphere gotten by gluing the section $S^{\prime \prime} \subset N^{\prime \prime}$ with the section $S$ of $V_{2 s}$ along the meridian of $F^{\prime \prime}=F$. Let $\Theta_{s+1}$ be the genus $(s+1)$ surface inside $M(s)$, gotten by resolving the intersection points of the union of $\Theta$ and $(s+1)$ distinct copies of $F$. We have $\left[\Theta_{s+1}\right]^{2}=0$ and $\left[\Theta_{s+1}\right] \cdot[F]=1$. Using the same kinds of argument as in the previous examples, we can show that each basic class $L$ is orthogonal to a codimension 4 subspace of $H_{2}\left(M_{n}(s)\right)$, namely,

$$
L=P D\left(a\left[\Gamma_{n+1}\right]+b\left[\Sigma_{2}\right]\right)+\left(c[F]+d\left[\Theta_{s+1}\right]\right) .
$$

From the adjunction inequality and the dimensional constraint $L^{2} \geq 3 \operatorname{sign}+2 e=$ $8 n$, we deduce that

$$
L= \pm P D\left(2\left[\Gamma_{n+1}\right]+2 n\left[\Sigma_{2}\right]+c[F]\right),
$$

where $c=0, \pm 2, \ldots, \pm 2 s$. Now we use the product formula for the Seiberg-Witten invariants in [MST] Theorem 3.1]. In the notation of [MST], $X=M_{n-1}(s), Y=W$, $C=\Sigma_{2}$, and $M=X \#_{C} Y=M_{n}(s)$.

First note that since $W$ is symplectic and $c_{1}(W)= \pm P D(2[T])$, we must have $S W_{W}( \pm P D(2[T]))= \pm 1$. Note also that in our case $H_{1}(\Sigma) \rightarrow H_{1}(W)$ is injective and this simplifies the product formula in [MST, Theorem 3.1]. In this case the knowledge of Seiberg-Witten invariants of $M_{0}(s)=M(s)$ and $W$ allows us to compute the $S W_{M_{n}(s)}(L)$ inductively, and we easily get:

Theorem 5.5. For each $n \geq 1, s \geq 1$ let the symplectic 4-manifold $M_{n}(s)$ be defined as in Section [3. Then the the basic classes of $M_{n}(s)$ are equal to $L=$ $\pm P D\left(2\left[\Gamma_{n+1}\right]+2 n\left[\Sigma_{2}\right]+c[F]\right)$, where $c=0, \pm 2, \ldots, \pm 2 s$. Furthermore

$$
S W_{M_{n}(s)}( \pm L)= \pm\left(\begin{array}{c}
2 s \\
s+c / 2
\end{array}\right) \text {. }
$$

Next let us determine the Seiberg-Witten basic classes $L$ of $Y(3, q) . Y(3,0)=V_{6}$ and it is well-known, see [FS3], that the set of basic classes are given by $L=$ $0, \pm 2 P D[F], \pm 4 P D[F]$ and $S W_{V_{6}}(P D(t[F]))= \pm\left(\begin{array}{c}4 \\ (4+t) / 2\end{array}\right)$. If $q>0$, then one can show, as in the previous examples, that every basic class $L$ is orthogonal 
to a codimension 2 subspace of $H_{2}(Y(3, q))$. The new wrinkle in the argument is due to a new $2 H$ summand coming from each copy of $W_{K}$. As before, we can find a basis for the corresponding subspace of $H_{2}(Y(3, q))$ consisting solely of tori with square 0 . (The new $2 H$ summand coming from $W_{K}$ arises when we glue together the 2-disks bounding the vanishing cycles of $F$ and appropriate cylinders in $\Sigma^{0} \times\left[\left(m \times S^{1}\right)-p t\right]$ to get two 2 -spheres of square -2 . The ends of cylinders are determined by the monodromy of the fibration $S^{3}(K) \rightarrow m$. This is analogous to the $2 H$ summand that we get when we fiber sum elliptic surfaces which contain cusp fibers. There is a canonical basis consisting of the two 2-spheres and two tori, which can be modified to a basis consisting of four tori of square 0 , after a suitable linear change of basis elements.) Thus $L=n P D\left[\Gamma_{q+1}\right]+m P D\left[\Sigma_{3}\right]$, where $\Gamma_{q+1}$ is a genus $(q+1)$ surface gotten by gluing a copy of the fiber $F$ in $V_{6}$ and $q$ copies of the fiber $T$ in $W_{K}$ along the $q$ meridional circles of $\Sigma_{3}$ (that all lie on the same copy of $F$ ) where the symplectic sums are taking place. The adjunction inequality implies $n=0, \pm 2, \pm 4$ and $m=0, \pm 2, \ldots, \pm 2 q$. Since $2 n m=L^{2} \geq 3 \operatorname{sign}+2 e=16 q$, we must have $L= \pm\left(4\left[\Gamma_{q+1}\right]+2 q\left[\Sigma_{3}\right]\right)$, and $S W_{Y(3, q)}(L)= \pm 1$.

Finally, we proceed to determine the SW-basic classes of $Y(s, q)$ for general $s \geq 3$. $Y(s, 0)=V_{2 s}$ and again we know that $L=0, \pm 2 P D[F], \ldots, \pm(2 s-2) P D[F]$ and $S W_{V_{2 s}}(P D(t[F]))= \pm\left(\begin{array}{c}2 s-2 \\ s-1+t / 2\end{array}\right)$. For $q>0$, there are only two basic classes, namely, $L= \pm P D\left((2 s-2)\left[\Gamma_{q+1}\right]+2 q\left[\Sigma_{s}\right]\right)$, where $\Gamma_{q+1}$ denotes a genus $(q+1)$ surface in $Y(s, q)$ gotten by gluing a copy of the fiber $F$ in $V_{2 s}$ and $q$ copies of the fiber $T$ in $W_{(s-2) K}$ along the $q$ meridional circles of $\Sigma_{s}$. Clearly $S W_{Y(s, q)}(L)= \pm 1$.

Proof of Theorem 1.3. First note that all the 4-manifolds $M_{n}(s)$ with $n \geq$ $0, s \geq 0$ contain a Gompf nuclei of the $K 3$ surface. Then we can use either the construction of [Sz2] or the more general construction in [FS2] to obtain nonsymplectic 4-manifolds with the same homotopy type as $M_{n}(s)$. Let us explain this below.

The obstruction to having a symplectic structure comes from a theorem of Taubes. Let $X$ be a closed symplectic 4-manifold with $b_{2}^{+}(X)>1$. Let $L_{1}, \cdots, L_{n}$ denote the basic classes of $X$, and let $\mathcal{B}(X) \subset H^{2}(X, \mathbf{R})$ denote the convex hull of $L_{1}, \cdots, L_{n}$. Then it follows from [T2 that the canonical class $c_{1}(X)$ lies in the boundary of $\mathcal{B}(X)$. In particular there is a basic class on the boundary of $\mathcal{B}(X)$ with Seiberg-Witten invariant \pm 1 .

Now take a quadratic form $Q$ as in Theorem 1.1 and let $M_{n}(s)$ denote the symplectic 4-manifold with $Q_{M_{n}(s)}=Q$. Let $F \hookrightarrow M_{n}(s)$ be a generic fiber inside a Gompf nuclei contained in $M_{n}(s)$. Following the construction of Sz2], take the fiber sum of $M_{n}(s)$ and the Kodaira-Thurston manifold $Y$, along the fiber $F \hookrightarrow$ $M_{n}(s)$ and a section $T_{1}$ of $Y$ and make a certain logarithmic transformation of multiplicity $p>1$ along the other torus $T_{2}$ defined in $\left[\mathrm{Sz} 2\right.$. Let $N_{n}(s, p)$ denote the resulting 4-manifold. It is clear (cf. [Sz2] or [FS2]) that $N_{n}(s, p)$ is simplyconnected, spin and $Q_{N_{n}(s, p)}=Q$. It follows from [Sz2] (cf. also [FS2]), that for every basic class $L$ of $N_{n}(s, p)$ that lies in the boundary of the convex hull $\mathcal{B}\left(N_{n}(s, p)\right)$, the Seiberg-Witten invariant is divisible by $p$. (In fact for these basic classes we have $S W(L)= \pm p$.) It follows that for all $p>1$ the 4-manifold $N_{n}(s, p)$ is not symplectic. Since $N_{n}(s, p)$ contains spheres with self-intersection -2 , it follows that the Seiberg-Witten invariant of $-N_{n}(s, p)$ vanishes. Now we can conclude that $N_{n}(s, p)$ doesn't admit symplectic structures with either orientations. On the other 
hand, since $N_{n}(s, p)$ is spin and has nontrivial Seiberg-Witten invariants, it follows that $N_{n}(s, p)$ is irreducible, and this finishes the proof of Theorem 1.3 .

\section{REFERENCES}

[D] S.K. Donaldson: An application of gauge theory to four-dimensional topology, J. Diff. Geom. 18 (1983), 279-315. MR 85c:57015

[FS1] R. Fintushel and R. Stern: Surgery in cusp neighborhoods and the geography of irreducible 4-manifolds, Invent. math. 117 (1994), 455-523. MR 95f:57040

[FS2] R. Fintushel and R. Stern: Knots, links and 4-manifolds, Invent. Math. 134 (1998), 363400. CMP 99:03

[FS3] R. Fintushel and R. Stern: Rational blowdowns of smooth 4-manifolds, J. Diff. Geom. 46 (1997), 181-235. MR 98j:57047

[G1] R.E. Gompf: Nuclei of elliptic surfaces, Topology 30 (1991), 479-511. MR 92f:57042

[G2] R.E. Gompf: A new construction of symplectic manifolds, Annals of Math. 142 (1995), 527-595. MR 96j:57025

[GM] R.E. Gompf and T.S. Mrowka: Irreducible 4-manifolds need not be complex, Annals of Math. 138 (1993), 61-111. MR 95c:57053

[Gr] M. Gromov: Partial Differential Relations, Springer-Verlag, Berlin, 1986. MR 90a:58201

[Ki] R. Kirby: Problems in low-dimensional topology, Geometric Topology, AMS/IP Stud. Adv. Math. 2.2, 35-473, Amer. Math. Soc., 1997. CMP 98:01

[KM] P.B. Kronheimer and T.S. Mrowka: The genus of embedded surfaces in the projective plane, Math. Res. Lett. 1 (1994), 797-804. MR 96a:57073

$[\mathrm{M}] \quad$ J.W. Morgan: The Seiberg-Witten equations and applications to the topology of smooth four-manifolds, Math. Notes 44, Princeton University Press, 1996. MR 97d:57042

[MSz] J.W. Morgan and Z. Szabó: Embedded genus-2 surfaces in four-manifolds, Duke Math. J., 89 (1997), 577-602. MR 98k:57048

[MST] J.W. Morgan, Z. Szabó and C.H. Taubes: A product formula for the Seiberg-Witten invariants and the generalized Thom conjecture, J. Diff. Geom. 44 (1996), 706-788. MR 97m:57052

[P] J. Park: Ph.D. Thesis, Michigan State University, 1995.

[St] A. Stipsicz: Elementary computations of Donaldson series, Turkish J. of Math. 19 (1995), 159-166. MR 96k:57031

[Sz1] Z. Szabó: Simply-connected irreducible 4-manifolds with no symplectic structures, Invent. Math. 132 (1998), 457-466. MR 99f:57033

[Sz2] Z. Szabó: Exotic 4-manifolds with $b_{2}^{+}=1$, Math. Res. Lett. 3, (1996), 731-741. MR 97j:57049

[Sz3] Z. Szabó: Exotic smooth structures on $(2 n+1)\left(S^{2} \times S^{2}\right)$, in preparation.

[Sz4] Z. Szabó: Irreducible four-manifolds with small Euler characteristics, Topology 35 (1996), 411-426. MR 97c:57021

[T1] C.H. Taubes: The Seiberg-Witten invariants and symplectic forms, Math. Res. Letters 1 (1994), 809-822. MR 95j:57039

[T2] C.H. Taubes: More constraints on symplectic manifolds from Seiberg-Witten invariants, Math. Res. Lett. 2, (1995), 9-13. MR 96a:57075

[Th] W. Thurston: Some simple examples of symplectic manifolds, Proc. Amer. Math. Soc. 55 (1976), 467-468. MR 53:6578

[Wi] E. Witten: Monopoles and four-manifolds, Math. Res. Lett. 1 (1994), 769-796. MR 96d:57035

Department of Mathematics, Princeton University, Princeton, New Jersey 08544

E-mail address: bahnpark@math.princeton.edu

Department of Mathematics, Princeton University, Princeton, New Jersey 08544

E-mail address: szabo@math.princeton.edu 